\title{
Time-dependent study of bistability in resonant-tunneling structures
}

\author{
E. Keçecioğlu and M. Cemal Yalabik \\ Department of Physics, Bilkent University, 06533 Ankara, Turkey
}

(Received 17 June 1998)

\begin{abstract}
A computational time-dependent study of the many-particle resonant-tunneling problem in the bistable regime is presented. The Schrödinger equation is solved directly, without the nonlinearizing mean-field-type approximations. The bistability then manifests itself in the form of instabilities, which occur as oscillations in the current-voltage characteristics. The time dependence of the wave function in the Schrödinger equation is studied by discretizing the energy spectrum and the time steps, for the two-, four-, and six-particle system. [S0163-1829(98)02348-0]
\end{abstract}

\section{INTRODUCTION}

The phenomenon of bistability arising in quantum transport systems is a common occurrence, both theoretically and experimentally. Possible practical uses of such switching behavior are obvious. ${ }^{1}$ A fundamental understanding of the phenomenon is also of interest.

One of the most studied systems displaying bistability in the current-voltage behavior is the double-barrier resonanttunneling structure. The earliest work on the switching times in these structures involves the use of Wigner function methods. ${ }^{2,3}$ The main interest in this early work is on the change in the current through the resonant structure when there is a large change in the potential difference (corresponding typically to the "peak" and the "valley" current values). Jensen and Buot ${ }^{4}$ used the Wigner function method to obtain an intrinsic "dynamical bistability" in the currentvoltage dependence: The current in the structure became nonuniform, and oscillated in time with a frequency dependent on the bias. The average value of the current is doublevalued as a function of the potential difference in this region of bias. It should be noted that the bistability appears with the inclusion of a self-consistent approximation to the interparticle interaction, which nonlinearizes the problem.

The first attempts to study the problem using a simulation of the time-dependent Schrödinger equation were made by Guo et $a .^{5}$ They analyzed the buildup and decay times of charge inside the double barriers through a simulation of the time development of a Gaussian wave packet incident on the structure. Mains and Haddad, ${ }^{6}$ studied the same problem utilizing the same method, using an ensemble of noninteracting particles. Their study, however, did not include the effects of the interparticle interaction, so that no bistability could be observed.

In this work, we will mainly be interested in a more fundamental aspect of this phenomenon, namely the appearance of the blatantly nonlinear response of the intrinsically linear quantum-mechanical system. Indeed, a full quantummechanical treatment of the system (including the interparticle interaction) may be viewed as a many-particle scattering problem, which should have a unique solution. It is an interesting question whether inclusion of a large number of particles is sufficient for the manifestation of bistability, or whether one would necessarily have to include dissipative effects (which would provide justification for the "nonlinearizing" mean-field-type self-consistent approximations).

In the next section, a simplified model for resonanttunneling structures and the interparticle interaction in the model are introduced. In the third section, a numerical approach, which is followed by the results of the analysis of the two-, four-, and six-particle system, is described. We conclude with the final section.

\section{A SIMPLE MODEL}

The potential energy of the double-barrier (DB) structure was simulated simply by two symmetric $\delta$ functions located at $x=-d$ and $x=d$, i.e., the potential energy is given by

$$
V(x)=\alpha[\delta(x-d)+\delta(x+d)],
$$

where $\alpha$ is the height of the barriers.

The particle-particle interaction is taken to be in the form

$$
V\left(x_{i}, x_{j}\right)= \begin{cases}\beta \delta\left(x_{i}-x_{j}\right) & \text { if }-d<x_{i}<d \\ 0 & \text { otherwise }\end{cases}
$$

Here $\beta$ is the strength of the interaction.

The model is shown in Fig. 1 schematically. Although this is admittedly a very simplified model, it does lead to bistability within a mean-field treatment, as seen in Fig. 2. The restriction of the interparticle interaction to the region between the barriers simplifies the analysis, and may be justified by the fact that the interaction dominates in this region due to the large value of the wave function in resonance.

The potential difference between the two-particle reservoirs (with an equal number of incident states) at the two

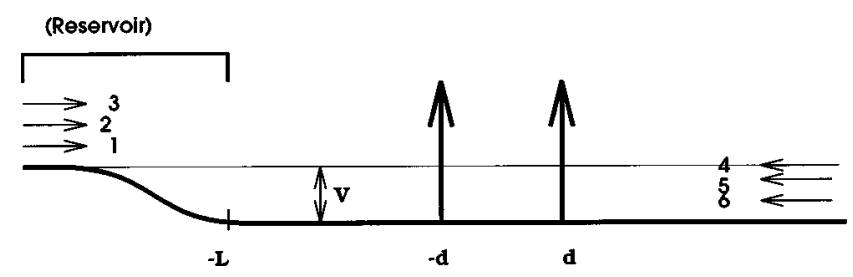

FIG. 1. A simple model for double-barrier resonant-tunneling structures. 


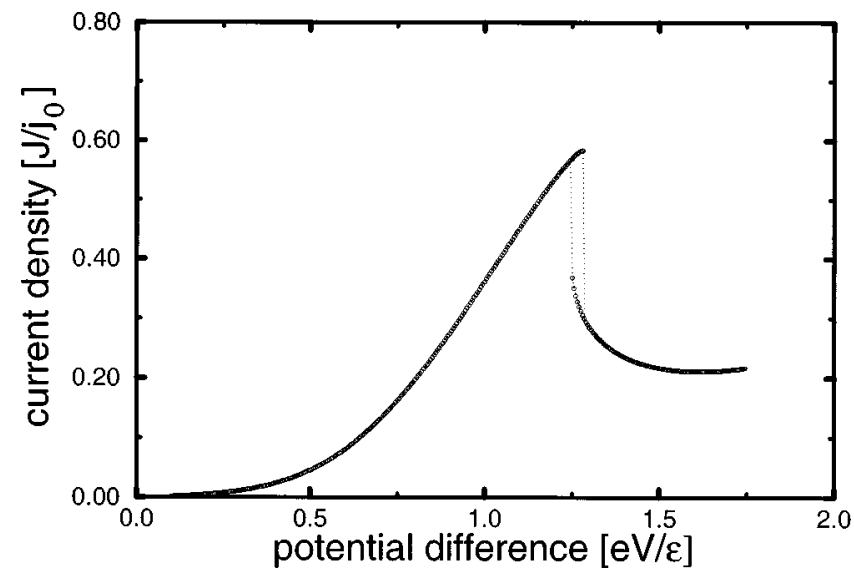

FIG. 2. Current versus applied bias for four particles in the mean-field approach. This result was obtained for $\beta$ $=0.0025 \hbar^{2} /\left(2 m d^{2}\right)$ and $\alpha=2.5 \alpha_{0}$.

ends of the structure is assumed to be due to a gradual shift in the zero level of the potential energy (the "band edge"'), as shown in the figure.

\section{THE METHOD}

The most general approach to the quantum transport problem may start with the $N$-particle, time-dependent Schrödinger equation:

$$
H \Psi\left(x_{1}, x_{2}, \ldots, x_{N}, t\right)=i \hbar \frac{\partial \Psi\left(x_{1}, x_{2}, \ldots, x_{N}, t\right)}{\partial t} .
$$

Here $H$ is the Hamiltonian, $\Psi$ is the $N$-particle timedependent wave function, and $\hbar$ is Planck's constant divided by $2 \pi$.
We separate the Hamiltonian in two parts:

$$
H=H_{0}+H_{1} \lambda(t)
$$

where $H_{0}$ is composed of terms including single-particle parts of the Hamiltonian and $H_{1}$ contains interaction between particles. The time dependence for $H_{1}$ is assumed. The system starts in some initial state with $\lambda(0)=0$ and develops adiabatically to the full Hamiltonian with $\lambda(\infty)=1$.

Note that the time-independent equation

$$
H_{0} \psi_{K}\left(x_{1}, x_{2}, \ldots, x_{N}, t\right)=E_{K} \psi_{K}\left(x_{1}, x_{2}, \ldots, x_{N}, t\right)
$$

is exactly solvable. Here $\psi_{K}\left(x_{1}, x_{2}, \ldots, x_{N}, t\right)$ denotes the many-particle wave function, including spin.

The analysis up to this point is exact. We now discretize the energy spectrum and the time variable in order to solve these equations numerically. We discretize the wave numbers of the incident single-particle states so that $K=k \Delta q$, with $k$ an integer. The $N$-particle state at any time $t$ may be expanded in terms of solutions of Eq. (5), so that

$$
\Psi=\sum_{K} F_{K}(t) \exp \left(-i E_{K} t / \hbar\right) \psi_{K}\left(x_{1}, x_{2}, \ldots\right)
$$

where $F_{K}(t)$ is the time-dependent expansion coefficients to be determined and $E_{K}=\Sigma_{q} \hbar^{2} q^{2} /(2 m)$ with $m$ the mass of the particles and $q$ the wave number corresponding to the single-particle states forming the determinental manyparticle wave function $K$.

Time-dependent coefficients obey the corresponding differential equation:

$$
\begin{gathered}
\sum_{K} e^{-i t\left(E_{K} / \hbar\right)} F_{K}(t) E_{K} \Psi_{K}+\sum_{i>j=1}^{N} V\left(x_{i}, x_{j}\right) \sum_{K} e^{-i t\left(E_{K} / \hbar\right)} F_{K}(t) \Psi_{K} \\
=i \hbar \sum_{K} \Psi_{K}\left[\left(\frac{-i E_{K}}{\hbar}\right) e^{-i t\left(E_{K} / \hbar\right)} F_{K}(t)+e^{-i t\left(E_{K} / \hbar\right)} \frac{\partial F_{K}}{\partial t}\right]
\end{gathered}
$$

which we integrate numerically using the discretization

$$
F_{Q}(t+\Delta t)=F_{Q}(t-\Delta t)-\frac{2 i \Delta t}{\hbar} \lambda(t) \sum_{k} H_{Q, K}^{1} \exp \left(-\frac{i\left(E_{K}-E_{Q}\right) t}{\hbar}\right) F_{K}(t) .
$$

Note that this equation is correct to order $(\Delta t)^{2}$ and preserves the time-reversal symmetry of the Schrödinger equation. This feature is important for the stability of the numerical integration.

\section{RESULTS}

When expanding the many-particle solution in terms of eigensolutions of $H_{0}$, an upper bound energy $E_{\text {upper }}$ is used, so that states whose energy is more than an amount $E_{\text {upper }}$ far from the incident state are excluded in the expansion.

The accuracy of the numerical solution depends of course on the coarseness of the discretizations (in $t$ and $E$ ) described above, and the number of states that are kept in the basis. We have typically kept 400-500 single-particle states in our analysis, and the adiabatic approach to the Hamiltonian was carried out in 15000 steps. Because of the upper bound energy $E_{\text {upper }}$ explained above, typically 4000 twoparticle states, 80000 four-particle states, and 400000 sixparticle states were kept in the basis. 


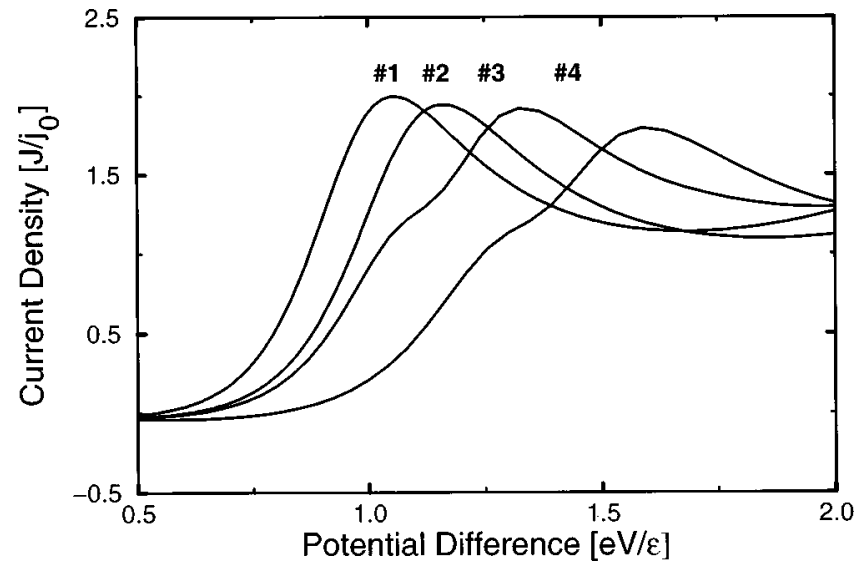

FIG. 3. Two-particle current versus potential difference for $\beta$ equal to $0,0.001,0.002$, and 0.0025 times $\epsilon$ for curves located 1 through 4 , respectively.

The simplicity of our model allows us to select and compute finite matrix elements "on the go," reducing computer storage requirements, however the computational task is still formidable. We have been able to perform computations up to six-particle systems with the computational resources available to us. An eight-particle computation for the current voltage curve with 15 data points takes more than two months with our computational resources.

In all the results that will be shown hereafter, $\alpha$ $=2.5 \alpha_{0}$, the energy is scaled with the quantity $\epsilon$ $=\hbar^{2} /\left(2 m d^{2}\right)$, with $2 d$ being the distance between the barriers. The time scale is scaled by $t_{0}=\hbar / \epsilon$ and the current by $j_{0}=\hbar /(2 m d)$.

The following values of the parameters were used in this work:

$$
\begin{gathered}
\Delta q=0.01 \sqrt{2 m \epsilon} / \hbar, \\
\Delta t=\hbar \pi / 15000 \epsilon, \\
\alpha=2.5 \alpha_{0},
\end{gathered}
$$

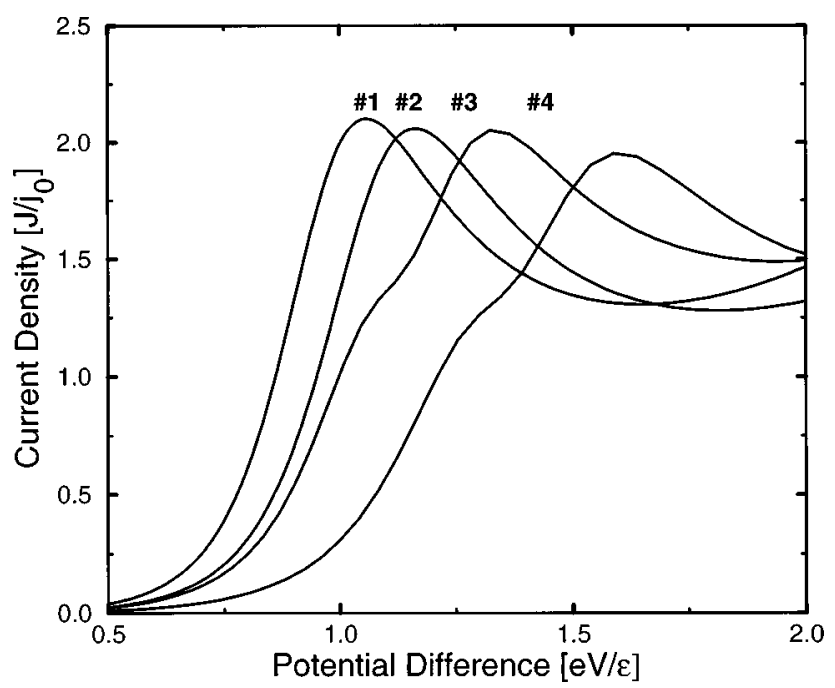

FIG. 4. Four-particle current versus potential difference. The labeling of the curves is as given in Fig. 3.

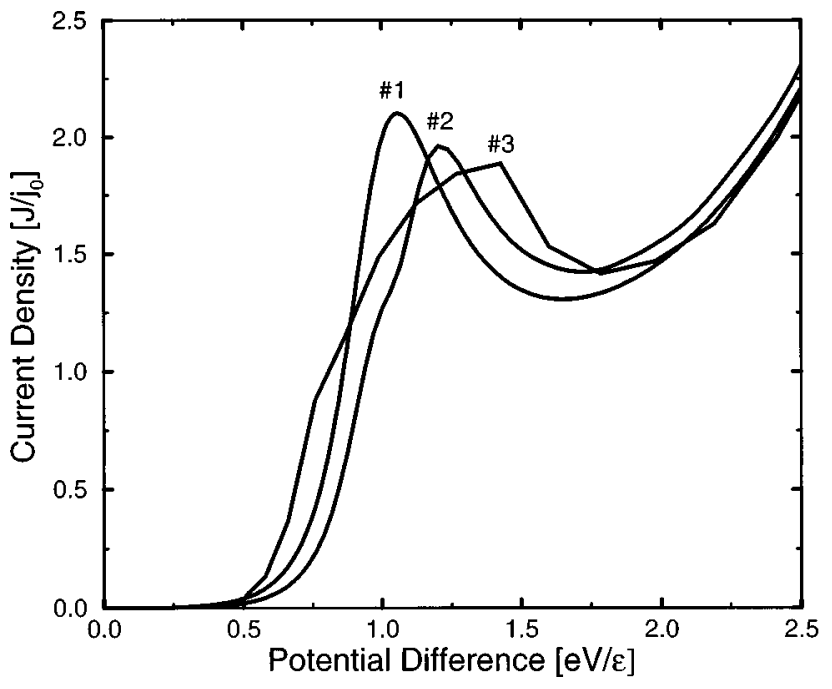

FIG. 5. Six-particle current versus potential difference for $\beta$ equal to $0,0.0015$, and 0.0025 times $\epsilon$ for curves located 1 through 4 , respectively.

$$
E_{\text {upper }}=4 \epsilon
$$

In Fig. 3, the current versus potential difference is shown for two particles. The same for four particles is shown in Fig. 4. The values $0,0.001,0.002$, and 0.0025 were used for the interaction constant $\beta / \epsilon$. The values of interaction were chosen on the basis of the mean-field approach, which leads to bistability for $\beta / \epsilon$ equal to 0.0025 . The magnitudes of the incident wave function are scaled appropriately as the number of particles in the computation is increased, consistent with the assumption of particle reservoirs on both sides of the geometry.

As the interaction constant increases, the current curves begin to broaden and some structure appears at energies lower than the peak energy. The broadening of the curves and the increase of the peak-current potential difference as the interparticle interaction increases is to be expected since

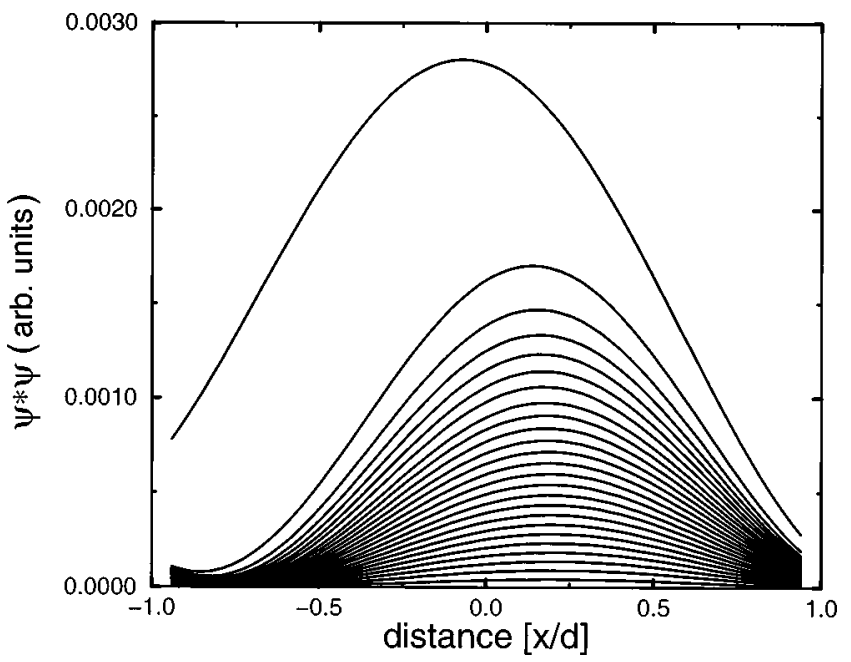

FIG. 6. Wave function between the barriers. The interaction constant increases from bottom curve to top curve. The lowest curve corresponds to $\beta=0.001 \epsilon$ and from bottom to top $\beta$ changes with $0.001 \epsilon$ intervals with $\beta=0.0025 \epsilon$ for the highest curve. Note the sudden change in the density. 


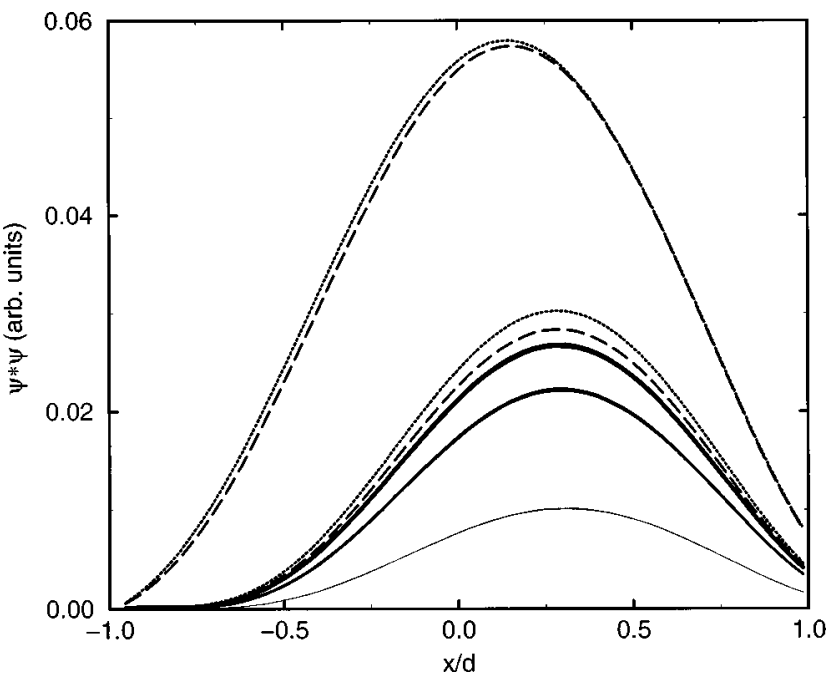

FIG. 7. Wave function between the barriers, the mean-field result. The interaction constant increases from bottom curve to top curve. The lowest curve corresponds to $\beta=0.001 \epsilon$ and the next lowest two curves correspond to $\beta=0.002 \epsilon$ and $\beta=0.0023 \epsilon$, respectively. The dashed curves are for $\beta=0.0024 \epsilon$ and the dotted curves are for $\beta=0.0025 \epsilon$. For different initial conditions meanfield solutions converge to the same final results for $\beta$ up to $0.0023 \epsilon$ and they converge to different bistable final results for bigger interaction constants. A comparison with our results in Fig. 6 shows that our method results in the lower curve for $\beta=0.0024 \epsilon$ and in the upper curve for $\beta=0.0025 \epsilon$. It may be concluded that the jump in Fig. 6 is due to instability of the solution.

the repulsive interparticle interaction results in a larger effective barrier in the geometry. The additional structure at lower energies may be interpreted as being due to the possible enhancement of transmission due to energy transfer between the particles.

In Fig. 5, six-particle results of current-voltage graphics are drawn for interaction strengths $0,0.0015$, and 0.0025 times $\epsilon$ in order of peak values from left to right.

As mentioned earlier, it is not possible to observe the bistability in a linear approach, but by examining the behavior of the wave function one may obtain information about

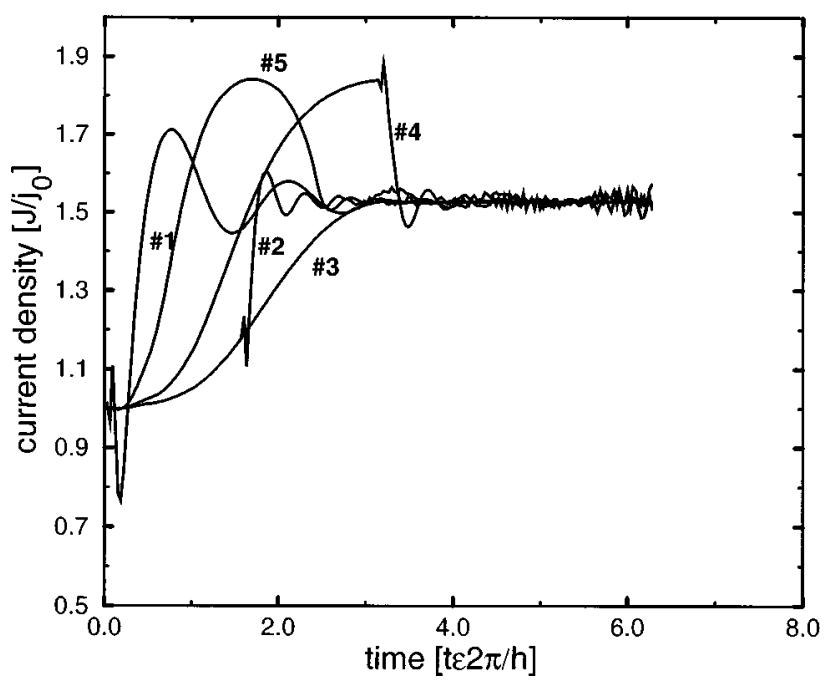

FIG. 8. Four-particle current versus time. Current calculated at some bias in the NDR region.

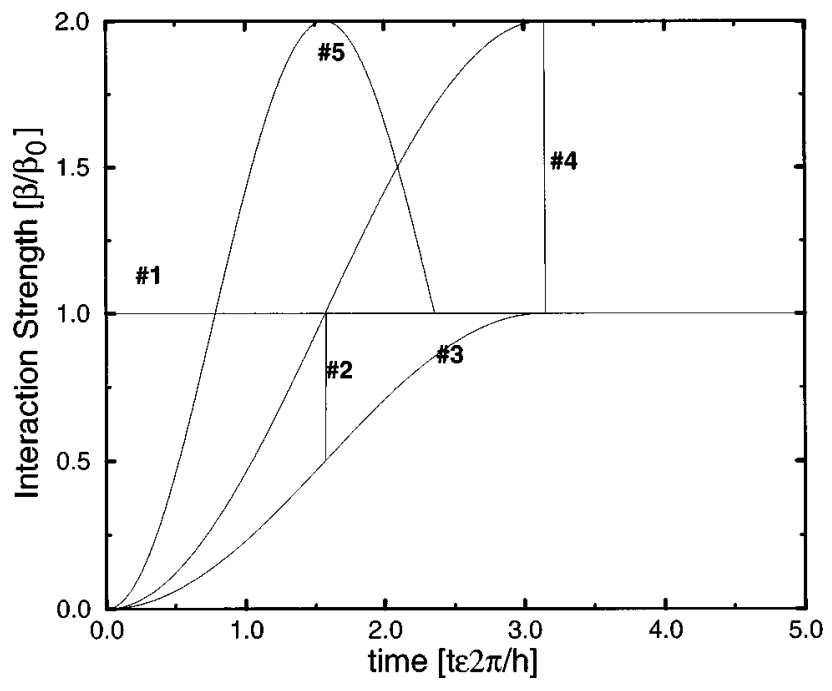

FIG. 9. Time dependence of the interaction strength corresponding to the preceding figure.

the mechanism. The magnitude of the wave function between the barriers is drawn in Fig. 6. Here the curves from bottom to top correspond to increasing interaction constants. The increment in the interaction constant is uniform throughout. It can be seen that at first the wave function changes uniformly as the strength of the interaction constant is changed, but at some point it rapidly changes to a very different form. This result may signal the onset of two solutions in the mean-field analysis, (see Fig. 7). In our computation, these "solutions" seem to indicate a single-particle effective potential in the form of two quantum wells. The quantumwell picture is enhanced by the appearance of oscillations in the current as a function of time in this regime.

Although the time dependence in our analysis is introduced artificially, it does serve to "prepare" the system in some pseudoinitial state (corresponding to different "initial guesses" in a self-consistent mean-field analysis). In Fig. 8 the time dependence of the current at a particular bias in the

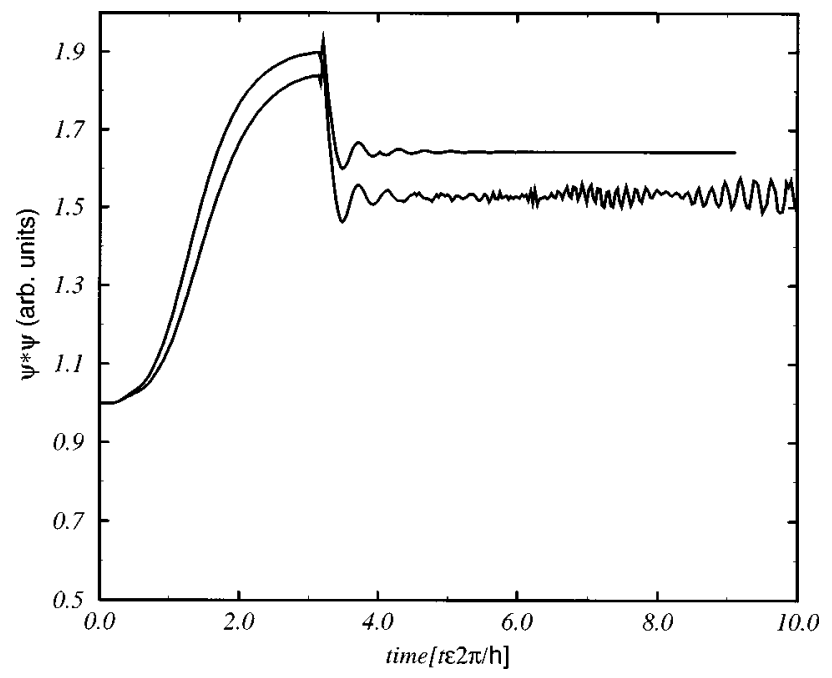

FIG. 10. Time dependence of the wave-function magnitude at some point between the barriers of the four-particle system for two different interaction constants. The upper curve is for $\beta$ equal to 0.0029 and the lower one is for 0.0025 times $\epsilon$. 


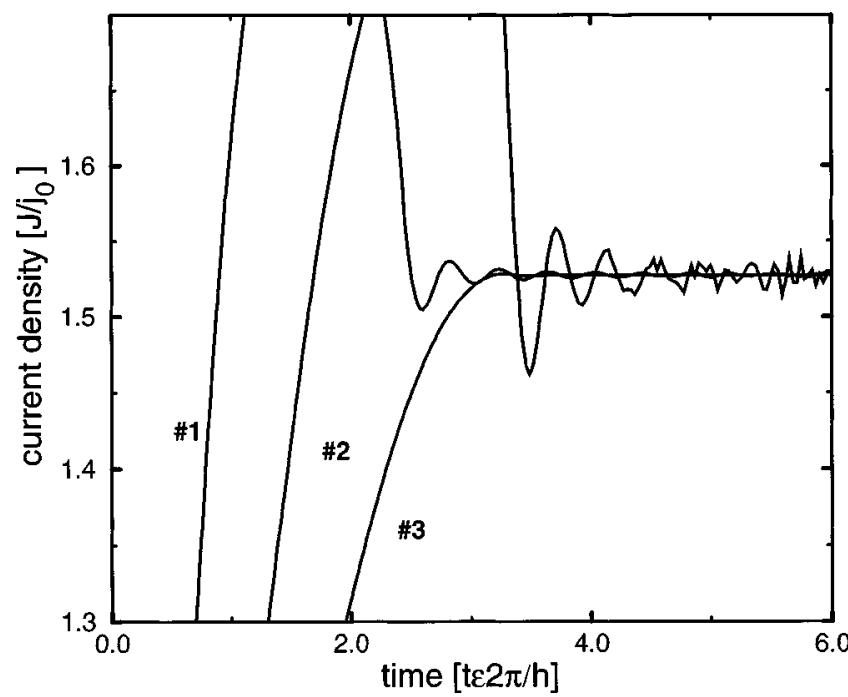

FIG. 11. Six-particle current versus time. Current calculated at some bias in the NDR region.

negative differential resistance (NDR) region is shown for different types of time dependence of the interaction strength. (This figure is for four particles.) Corresponding time dependences are shown in Fig. 9. It is seen from Fig. 8 that there are instabilities in the solution, which may be interpreted as transitions between coupled quasistable solutions of the system.

In Fig. 10, time dependencies of the wave-function magnitude at some point between the barriers for two different interaction strengths are drawn. Corresponding values of the strengths are 0.0029 and 0.0025 times $\epsilon$ from top to bottom curve, respectively. It is clear that the oscillations that are seen in the smaller interaction no longer exist in the larger interaction. The lower curve is the current at a bistable value, while the upper curve is the current at an interaction slightly above the bistable value.

In Fig. 11 and Fig. 12, oscillations and corresponding time dependence of the interaction strengths are drawn for six particles in the NDR region for an interaction strength where bistability is expected. Results are similar for the twoand four-particle cases.

\section{CONCLUSION}

A computational approach based on discretizing the spectrum of the noninteracting Hamiltonian for the study of

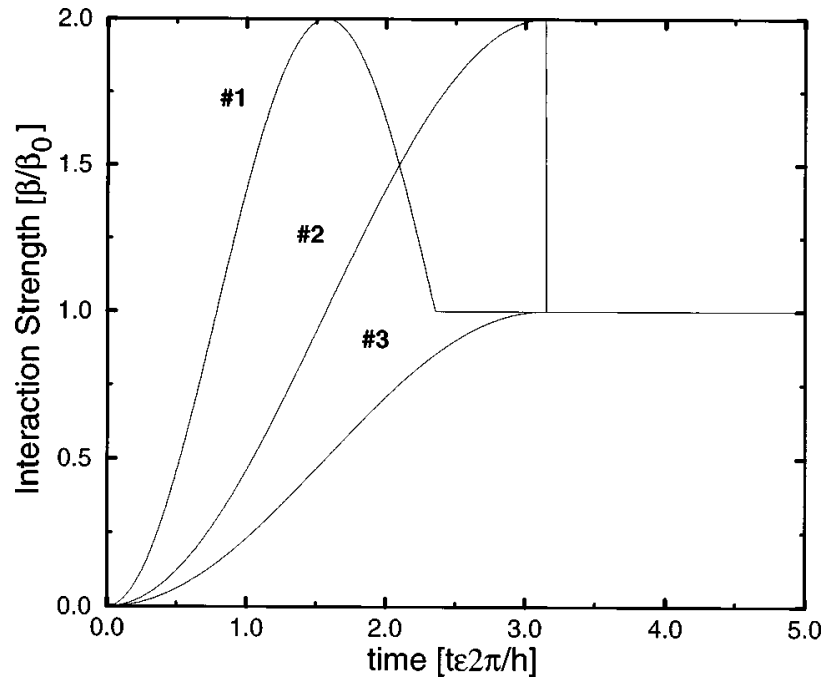

FIG. 12. Time dependence of the interaction strength corresponding to Fig. 11.

quantum transport in small systems in the many-body picture was proposed. The method mainly depends on discretizing the spectrum of the noninteracting Hamiltonian, expanding the solution in terms of this spectrum and integrating the time dependence of the wave function. The computational method is a linear treatment. The analysis is "computationally exact" apart from an energy cutoff and the discretizations.

A simple model for analyzing the bistability in doublebarrier resonant-tunneling structures was constructed. Although the model is very simple, it does nevertheless exhibit bistability in a mean-field treatment. The computational effort is still formidable due to the large number of unperturbed many-particle states that must be kept.

The simulations were performed for two, four, and six interacting particles. The behavior of the system under the conditions in which bistability is expected (from a meanfield approximation) is studied. Oscillations of the currentvoltage characteristics in time were observed in this regime. This was interpreted as transitions between coupled quasistable solutions of the system. To observe the oscillations, different types of time dependencies for the interparticle interactions were used. It was shown that outside the bistable region (expected from the mean-field approach) similar oscillations do not exist.
${ }^{1}$ T. C. G. L. Sollner, P. E. Tannenwald, D. D. Peck, and W. D. Goodhue, Appl. Phys. Lett. 45, 1319 (1984).

${ }^{2}$ N. C. Kluksdahl, A. M. Kriman, D. K. Ferry, and C. Ringhofer, IEEE Electron Device Lett. 9, 457 (1988).

${ }^{3}$ K. L. Jensen and F. A. Buot, J. Appl. Phys. 65, 5248 (1989).
${ }^{4}$ K. L. Jensen and F. A. Buot, Phys. Rev. Lett. 66, 1078 (1991).

${ }^{5}$ H. Guo, K. Diff, G. Neofotistos, and J. D. Gunton, Appl. Phys. Lett. 53, 131 (1988).

${ }^{6}$ R. K. Mains and G. I. Haddad, J. Appl. Phys. 70, 7638 (1991). 